\title{
RESULTS OF SEMIGROUP OF LINEAR OPERATOR IN SPECTRAL THEORY
}

\author{
A. Y. AKINYELE ${ }^{1, *}$, O. Y. SAKA-BALOGUN ${ }^{2}$, M. A. GANIYU ${ }^{3}$ \\ ${ }^{1}$ Department of Mathematics, University of Ilorin, Ilorin, Nigeria \\ ${ }^{2}$ Department of Mathematical and Physical Sciences, Afe Babalola University, Ado-Ekiti, Nigeria \\ ${ }^{3}$ Department of Physical Sciences, Al-hikmah University, Ilorin, Nigeria \\ *Corresponding author: olaakinyele04@gmail.com
}

Received Dec. 11, 2020

\begin{abstract}
АвsтRACт. This paper consists of results $\omega$-order preserving partial contraction mapping ( $\omega$ $\left.O C P_{n}\right)$ as a Semigroup of a linear operator in spectral theory. We consider $A \in \omega-O C P_{n}$ as the infinitesimal generator of a $C_{0}$-semigroup using the Spectral Mapping Theorem (SMT) to establish the relationships between the spectrum of $A$ and the spectrum of each of the operators $T(t), t \geq 0$.

2010 Mathematics Subject Classification. 06F15; 06F05; $20 \mathrm{M} 05$.
\end{abstract}

Key words and phrases: spectrum; $\omega-O C P_{n} ; C_{0}$-semigroup; Resolvent.

\section{INTRODUCTION}

The study of spectral theory in mathematics is an inclusive term for theories extending the eigenvector and eigenvalue theory of a single square matrix to a much broader theory of the structure of operators in a variety of mathematical spaces. Assume $X_{n} \subseteq X$ is a finite set, $(T(t))_{t \geq 0}$ the $C_{0}$-semigroup, $\omega-O C P_{n}$ the $\omega$-order preserving partial contraction mapping, $M_{m}$ be a matrix, $L(X)$ be a bounded linear operator on $X, P_{n}$ a partial transformation semigroup, $\rho(A)$ a resolvent set, $\sigma(A)$ a spectrum of $A$ and $A \in \omega-O C P_{n}$ is a generator of $C_{0}$-semigroup. This paper consist of results of $\omega$-preserving partial contraction mapping generating a spectral results. Balakrishnan [1], deduced some fractional powers of closed operators. Banach [2], established and introduced the concept of Banach spaces. Engel and Nagel [3], obtained oneparameter semigroup for linear evolution equations. Greiner et al. [4], proved some results on

DOI: $10.28924 / \mathrm{APJM} / 8-8$ 
the spectral bond generator of semigroup of positive operators. Hasegawa [5], presesented some results on the convergence of resolvents of operators. Pazy [6], introduced semigroup of linear operators and applications to partial differential equations. Rauf and Akinyele [7], introduced $\omega$-order-preserving partial contraction mapping and established its properties, also in [8], Rauf et al. deduced some results of stability and spectra properties on semigroup of linear operator. Slemrod [9], explained asymptotic behavior of $C_{0}$-semigroup as determined by the spectrum of the generator. Vrabie [10], proved some results of $C_{0}$-semigroup and its applications. Yosida [11], established and proved some results on differentiability and representation of one-parameter semigroup of linear operators.

\section{Preliminaries}

Definition 2.1 ( $C_{0}$-Semigroup) [10]

A $C_{0}$-Semigroup is a strongly continuous one parameter semigroup of bounded linear operator on Banach space.

Definition $2.2\left(\omega-O C P_{n}\right)[7]$

A transformation $\alpha \in P_{n}$ is called $\omega$-order-preserving partial contraction mapping if $\forall x, y \in \operatorname{Dom} \alpha$ : $x \leq y \Longrightarrow \alpha x \leq \alpha y$ and at least one of its transformation must satisfy $\alpha y=y$ such that $T(t+s)=T(t) T(s)$ whenever $t, s>0$ and otherwise for $T(0)=I$.

Definition 2.3 (Resolvent Set) [3]

We define the resolvent set of $A$ denoted by $\rho(A)$ set of all $\lambda \in \mathbb{C}$ such that $\lambda \mathrm{I}$ - $\mathrm{A}$ is one-to-one with range equal to $X$

\section{Definition 2.4 (Spectrum) [10]}

The spectrum of $A$ denoted by $\sigma(A)$ is defined as the complement of the resolvent set.

\section{Example 1}

Let $X$ be the Banach space of Continuous function on $[0,1]$ which are equal to zero at $x=1$ with the supremum norm. Define

$$
(T(t) f)(x)=\left\{\begin{array}{c}
f(x+t) \text { if } x+t \leq 1 \\
0 \text { if } x+t>1
\end{array}\right.
$$

$T(t)$ is obviously a $C_{0}$-Semigroup of Contractions on $X$. Its infinitesimal generator $A \in \omega-O C P_{n}$ is given by

$$
D(A)=\left\{f: f \in C^{\prime}([0,1]) \cap X_{1} f^{\prime} \in X\right\}
$$

and

$$
A f=f^{\prime} \text { for } f \in D(A)
$$


one checks easily that for every $\lambda \in \mathbb{C}$ and $g \in X$ the equation $\lambda f-f^{\prime}=g$ has a unique solution $f \in X$ given by

$$
f(t)=\int_{t}^{1} e^{\lambda(t-s)} g(s) d s .
$$

Therefore $\sigma(A)=\phi$. on the other hand, since for every $t \geq 0, T(t)$ is a bounded linear operator, $\sigma(T(t)) \neq \phi$ for all $t \geq 0$ and the relation $\sigma(T(t))=\exp \{t \sigma(A)\}$ does not hold for any $t \geq 0$.

\section{Theorem 2.1(Hille-Yoshida) []}

A linear operator $A: D(A) \subseteq X \rightarrow X$ is the infinitesimal generator for a $C_{0}$-semigroup of contraction if and only if

i. $A$ is densely defined and closed; and

ii. $(0,+\infty) \subseteq \rho(A)$ and for each $\lambda>0$, we have

$$
\|R(\lambda, A)\|_{L(X)} \leq \frac{1}{\lambda}
$$

\section{Main Results}

In this section, differentiable and analytic results on $\omega-O C P_{n}$ in semigroup of linear operator $\left(C_{0}\right.$-semigroup) were established:

\section{Theorem 3.1}

This section presents results of spectral theory generated by $\omega-O C P_{n}$ which were established on semigroup of linear operator:

\section{Theorem 3.1}

Assume $T(t)$ is a $C_{0}$-semigroup and let $A \in w-O C P_{n}$ be its infinitesimal generator. If

$$
B_{\lambda}(t) x=\int_{0}^{t} e^{\lambda(t-s)} T(s) x d s
$$

then

$$
(\lambda I-A) B_{\lambda}(t) x=e^{\lambda t} x-T(t) x \text { for every } x \in X
$$

and

$$
B_{\lambda}(t)(\lambda I-A) x=e^{\lambda t} x-T(t) x \text { for every } x \in D(A)
$$

and $A \in w-O C P_{n}$.

Proof: 
For every fixed $\lambda$ and $t, B_{\lambda}(t)$ defined by (3.1) is a bounded linear operator on X. Moreover, for every $x \in X$ and $A_{1} B \in w-O C P_{n}$ we have

$$
\begin{array}{r}
\frac{T(h)-1}{h} B_{\lambda}(t) x=\frac{e^{\lambda h}-1}{h} \int_{h}^{t} e^{\lambda(t-s)} T(s) x d s \\
+\frac{e^{\lambda h}}{h} \int_{t}^{t+h} e^{\lambda(t-s)} T(s) x d s-\frac{1}{h} \int_{0}^{h} e^{\lambda(t-s)} T(s) x d s .
\end{array}
$$

As $h \rightarrow 0$ in the right-hand side of (3.4) converges to $\lambda B_{\lambda}(t) x+T(t) x-e^{\lambda t} x$ and consequently $B_{\lambda}(t) x \in D(A)$ and

$$
A B_{\lambda}(t) x=\lambda B_{\lambda}(t) x+T(t) x-e^{\lambda t} x
$$

which implies (3.2). From the definition of $B_{\lambda}(t)$, it is clear that for $x \in D(A)$ and $A \in w-O C P_{n}$, we have

$$
A B_{\lambda}(t) x=B_{\lambda}(t) A x
$$

which implies

$$
B_{\lambda}(t)(\lambda I-A) x=e^{\lambda t} x-T(t) x .
$$

Hence, the prove is completed.

\section{Theorem 3.2}

Let $T(t)$ be a $C_{0}$-semigroup and $A \in w-O C P_{n}$ be the infinitesimal generator of semigroup of linear operator. Then

$$
\sigma(T(t)) \supset e^{t \sigma(A)} \text { for } t \geq 0
$$

\section{Proof}

Let $e^{\lambda t} \in \rho(T(t))$ and let $Q=\left(e^{\lambda t} I-T(t)\right)^{-1}$. The operators $B_{\lambda}(t)$, defined by (3.1), and $\mathrm{Q}$ clearly commute. From (3.2) and (3.3) we deduce

$$
(\lambda I-A) B_{\lambda}(t) Q x=x
$$

for every $x \in X$ and $A \in w-O C P_{n}$, and

$$
Q B_{\lambda}(t)(\lambda I-A) x=x
$$

for every $x \in D(A)$ and $A \in w-O C P_{n}$.

Since $B_{\lambda}(t)$ and $\mathrm{Q}$ commute, we also have

$$
B_{\lambda}(t) Q(\lambda I-A) x=x
$$


for every $x \in D(A)$ and $A \in w-O C P_{n}$.

Therefore, $\lambda \in \rho(A), B_{\lambda}(t) Q=(\lambda I-A)^{-1}=R(\lambda ; A)$ and $\rho(T(t)) \subset \exp (t \rho(A))$ which implies

$$
\sigma(T(t)) \supset e^{t \sigma(A)}
$$

we recall that the spectrum of $A$ consists of three mutually exclusive part; the point spectrum $\sigma_{p}(A)$, the continuous spectrum $Q_{c}(A)$ and the residual spectrum $\sigma_{t}(A)$. These are defined as follows: $\lambda \in \sigma_{p}(A)$ if $\lambda I-A$ is not one-to-one, $\lambda \in \sigma_{c}(A)$ if $\lambda I-A$ is one-to-one and its range is not dense in $\mathrm{X}$. From these definitions it is clear that $\sigma_{p}(A), \sigma_{c}(A)$ and $\sigma_{t}(A)$ are mutually exclusive and that their union is $\sigma(A)$, therefore the proof is achieved.

\section{Theorem 3.3}

Suppose $T(t)$ is a $C_{0}$-semigroup and let $A \in w-O C P_{n}$ be its infinitesimal generator. Then

$$
e^{t \sigma_{p}(A)} \subset \sigma_{p}(T(t)) \subset e^{t \sigma_{p}(A)} \cup\{0\} .
$$

More precisely if $\lambda \in \sigma_{p}(A)$ then $e^{\lambda t} \in \sigma_{p}(T(t))$ and if $e^{\lambda t} \in \sigma_{p}(T(t))$ then there exists a $k, k \in \mathbb{N}$ such that $\lambda_{k}=\lambda+2 \pi i k / t \in \sigma_{p}(A)$.

\section{Proof:}

Suppose $\lambda \in \sigma_{p}(A)$ then there is $x_{0} \in D(A), x_{0} \neq 0$, such that $(\lambda I-A) x_{0}=0$. From (3.3) it then follows that $\left(e^{\lambda t} I-T(t)\right) x_{0}=0$ and therefore $e^{\lambda t} \in \sigma_{p}(T(t))$ which proves the first inclusion. To prove the second inclusion, let $e^{\lambda t} \in \sigma_{p}(T(t))$ and let $x_{0} \neq 0$ satisfy $\left(e^{\lambda t} I-T(t)\right) x_{0}=0$. This implies that the continuous function $s \rightarrow e^{-\lambda s} T(s) x_{0}$ is periodic with period $t$ and since it does not varnish identically and one of its Fourier Coefficients must be different from zero. Therefore is a $k, k \in \mathbb{N}$ such that

$$
x_{k}=\frac{1}{t} \int_{0}^{t} e^{-(2 \pi i k / t) s}\left(e^{-\lambda s} T(s) x_{0}\right) d s \neq 0
$$

We will show that $\lambda_{k}=\lambda+2 \pi i k / t$ is an eigen value of $A$. Let $\|T(t)\| \leq M e^{w t}$. For $\operatorname{Re} \mu>w$ we have

$$
\begin{aligned}
R(\mu ; A) x_{0} & =\int_{0}^{\infty} e^{-\mu s} T(s) x_{0} d s=\sum_{n=0}^{\infty} \int_{n t}^{(n+1) t} e^{-\mu s} T(s) x_{0} d s \\
& =\sum_{n=0}^{\infty} e^{n(\lambda-\mu) t} \int_{0}^{t} e^{-\mu s} T(s) x_{0} d s \\
& =\left(1-e^{(\lambda-\mu) t}\right)^{-1} \int_{0}^{t} e^{-\mu s} T(s) x_{0} d s
\end{aligned}
$$

where we used the periodicity of $e^{-\lambda s} T(s) x_{0}$. The integral on the right-hand side of (3.12) is clearly an entire function and therefore $R(\mu ; A) x_{0}$ can be extended by (3.12) to a meromorphic 
function with possible poles at

$$
\lambda_{n}=\lambda+2 \pi i n / t, n \in \mathbb{N} .
$$

Using (3.12), it is easy to show that

$$
\lim _{\mu \rightarrow \lambda_{k}}\left(\mu-\lambda_{k}\right) R(\mu: A) x_{0}=x_{k}
$$

and

$$
\lim _{\mu \rightarrow \lambda_{k}}\left(\lambda_{k}-A\right)\left[\left(\mu-\lambda_{k}\right) R(\mu ; A) x_{0}\right]=0
$$

From the closedness of $A$ and (3.13), (3.14) it follows that $x_{k} \in D(A)$ and that $\left(\lambda_{k} I-A\right) x_{k}=0$, i.e; $\lambda_{k} \in \sigma_{p}(A)$. Hence, the proof is complete.

\section{Theorem 3.4}

Assume $T(t)$ is a $C_{0}$-semigroup and let $A \in w-O C P_{n}$ be the infinitesimal generator of semigroup $\mathrm{f}$ linear operator. Then

(i) if $\lambda \in \sigma_{t}(A)$ and none of the $\lambda_{n}=\lambda+2 \pi i n / t, n \in \mathbb{N}$ is in $\sigma_{p}(A)$ then $e^{\lambda t} \in \sigma_{t}(T(t))$; and

(ii) if $e^{\lambda t} \in \sigma_{t}(T(t))$ then none of the $\lambda_{n}=\lambda+2 \pi i n / t, n \in \mathbb{N}$ is in $\sigma_{p}(A)$ and there exists a $K, K \in \mathbb{N}$ such that $\lambda_{k} \in \sigma_{t}(A)$.

\section{Proof:}

If $\lambda \in \sigma_{t}(A)$ then there is a $x^{*} \in X^{*}, x^{*} \neq 0$, such that $<x^{*},(\lambda I-A) x>=0$ for all $x \in D(A)$. From (3.2) it then follows that

$$
<x^{*},\left(e^{\lambda t} I-T(t)\right) x>=0
$$

for all $x \in X$ and therefore the range of $e^{\lambda t} I-T(t)$ is not dense in X. If $e^{\lambda t} I-T(t)$ is not one-to-one then by Theorem 3.3, there is a $k \in \mathbb{N}$ such that $\lambda_{k} \in \sigma_{p}(A)$ contradicting our assumption that $\lambda_{n} \notin \sigma_{p}(A)$. Therefore $e^{\lambda t} I-T(t)$ is one-to-one and $e^{\lambda t} \in \sigma_{t}(T(t))$ which concludes the proof of $(i)$.

To prove (ii), we note first that if for some $\mathrm{k}, \lambda_{k}=\lambda+2 \pi i k / t \in \sigma_{p}(A)$ then by Theorem 3.3 $e^{\lambda t} \in \sigma_{p}(T(t))$ contradicting the assumption that $e^{\lambda t} \in \sigma_{t}(T(t))$. It suffices therefore to show that for some $k \in \mathbb{N}, \lambda_{k} \in \sigma_{t}(A)$. This follows at once if we show that $\left\{\lambda_{n}\right\} \subset \rho(A) \cup \sigma_{p}(A)$ is impossible. From (3.3) we have

$$
\left(e^{\lambda_{n} t} I-T(t)\right) x=B_{\lambda_{n}}(t)\left(\lambda_{n} I-A\right) x
$$

for $x \in D(A), A \in w-O C P_{n}$ and $n \in \mathbb{N}$.

Since by our assumption $e^{\lambda t}=e^{\lambda_{n} t} \in \sigma_{t}(T(t))$, the left-hand side of (3.15) belongs to a fixed 
non dense linear subspace $\mathrm{Y}$ of $\mathrm{X}$. On the other hand if $\lambda_{n} \in \rho(A) \cup \sigma_{c}(A)$ then the range of $\lambda_{n} I-A$ is dense in $\mathrm{X}$ which implies by (3.15) that the range of $B_{\lambda_{n}}(t)$ belongs to $\mathrm{Y}$ for every $n \in \mathbb{N}$. Writing the Fourier series of the continuous function $e^{-\lambda s} T(s) x$ we have

$$
e^{-\lambda s} T(s) x \sim \frac{1}{t} \sum_{n=-\infty}^{\infty} e^{(2 \pi i n / t)} B_{\lambda_{n}}(t) x
$$

and each term on the right-hand side of (3.16) belongs to Y. As in the classical numerical case the series (3.16) is summable to $e^{-\lambda s} T(s) x$ for $0<s<t$ and therefore for $0<s<$ $t, e^{-\lambda s} T(s) x \in Y$. Letting $s \rightarrow 0$ it follows that every $x \in D(A)$ satisfies $x \in \bar{Y}$ which is impossible since $\bar{Y}$ is a proper closed subspace of $\mathrm{X}$ and $\mathrm{D}(\mathrm{A})$ is dense in $\mathrm{X}$ and the prove is achieved.

\section{Theorem 3.5}

Suppose $T(t)$ is $C_{0}$-semigroup and let $\mathrm{A}$ be its infinitesimal generator. If $\lambda \in \sigma_{c}(A)$ and if none of the $\lambda_{n}=\lambda+2 \pi i n / t$ in $\sigma_{p}(A) \cup \sigma_{r}(A)$ then $e^{\lambda t} \in \sigma_{c}(T(t))$.

\section{Proof:}

From 3.2 it follows that if $\lambda \in \sigma_{c}(A)$ then $e^{\lambda t} \in \sigma(T(t))$.

If $e^{\lambda t} \in \sigma_{p}(T(t))$, then by Theorem 3.3 some $\lambda_{k} \in \sigma_{p}(A)$ and therefore $e^{\lambda t} \notin \sigma_{p}(T(t))$.

Similarly if $e^{\lambda t} \in \sigma_{r}(T(t))$ then some $\lambda_{k} \in \sigma_{r}(A)$ and again $e^{\lambda t} \notin \sigma_{r}(T(t))$.

Hence, the prof is complete.

\section{Conclusion}

In this paper, it has been established that $\omega$-order preserving partial contraction mapping $\left(\omega-O C P_{n}\right)$ generates results on spectral theory as a semigroup of linear operator.

\section{REFERENCES}

[1] A. V. Balakrishnan, Fractional powers of closed operators and the semigroup generated by them, Pac. J. Math. 10 (1960), 419-437.

[2] S. Banach, Surles Operation Dam Les Eusembles Abstracts et lear Application Aus Equation integrals, Fund. Math. 3 (1922), 133-181.

[3] K. Engel, R. Nagel, One-parameter Semigroups for Linear Equations, Graduate Texts in Mathematics, 194, Springer, New York, (2000).

[4] G. Greiner, J. Voigt, and M. Wolf, On the Spectral Bond Generator of Semigroups of Positive Operators, J. Oper. 5 (1981), 245-256.

[5] M. Hasegawa, On the Convergence of Resolvents of Operators, Pac. J. Math. 21 (1967), 35-47. 
[6] A. Pazy, Semigroup of Linear Operators And Applications to Partial Differential Equations, Applied Mathematical Sciences, 44, Springer Verlag, New York, Berlin Heidelberg, Tokyo, (1983).

[7] K. Rauf, A. Y. Akinyele, Properties of $\omega$-Order-Preserving Partial Contraction Mapping and its Relation to $C_{0}$-semigroup, Int. J. Math. Comput. Sci. 14 (2019), 61-68.

[8] K. Rauf, A. Y. Akinyele, M. O. Etuk, R. O. Zubair, M. A. Aasa, Some Result of Stability and Spectra Properties on Semigroup of Linear Operator, Adv. Pure Math. 9 (2019), 43-51.

[9] M. Slemrod, Asymptotic Behaviour of $C_{0}$-semigroup as Determined by the Spectrum of the Generator, Indiana Univ. Math. J. 25 (1976), 783-792.

[10] I. I. Vrabie, $C_{0}$ - Semigroup And Application, Mathematics Studies, 191, Elsevier, North-Holland, (2003).

[11] K. Yosida, On The Differentiability and Representation of One-Parameter Semigroups of Linear Operators, J. Math. Soc. Japan, 1 (1948), 15-21. 